\title{
THE HAEMOPHILIC PSEUDOTUMOUR OR HAEMOPHILIC SUBPERIOSTEAL HAEMATOMA
}

\author{
J. A. Fernandez de Valderrama, ${ }^{*}$ Madrid, Spain, and \\ J. M. Matthews, † Oxford, England \\ From the Nuffield Orthopaedic Centre and the Churchill Hospital, Oxford
}

Starker in 1918 described in detail the morbid anatomy of two subperiosteal haematomata in a fourteen-year-old haemophilic boy. The larger of these involved the femur and was at first diagnosed as an osteosarcoma. Echternacht (1943) described a similar lesion in the tibia of a thirteen-year-old haemophiliac.

The term haemophilic pseudotumour is used to describe a progressive cystic swelling involving muscle, produced by recurrent haemorrhage and accompanied by radiographic evidence of bone involvement.

Abell and Bailey (1960) reviewed the condition, found seventeen cases in the literature before 1959, and added two. Two of three cases described by Fraenkel, Taylor and Richards in 1959, and one of those described by Hall, Handley and Webster in 1962, conform to the same type. The number of pseudotumours so far reported seems to be twenty-two.

A fairly clear distinction may be drawn between those haemophilic cysts which are confined to muscle and have little or no effect on adjacent bone, and the pseudotumours which affect muscle and are notable for the associated bone changes. Radiography shows evidence of mingled areas of bone destruction and new bone formation. This, together $\mathrm{with}$ the soft-tissue swelling-which may be severe-presents a clinical picture which may be indistinguishable from that of a malignant tumour of bone. Whereas the history of haemophilia may be suggestive, osteosarcoma has been reported in a haemophiliac.

In this paper we describe a haemophiliac who had a cyst of the thigh, treated by amputation. The available evidence shows that the cyst was subperiosteal in origin. The patient (P. M.) has previously been referred to by Crock and Boni (1960).

\section{CASE REPORT}

The patient, a man of twenty-four, was a severely affected haemophiliac whose blood completely lacked antihaemophilic globulin (Factor VIII, AHG). The history of many severe haemorrhages at various sites included one in the right calf at the age of nine, which produced gangrene of the foot necessitating below-knee amputation.

By the age of seven he had developed a 90 degrees flexion deformity of the left knee after a haemarthrosis. An unsuccessful attempt to manipulate the knee a year later caused a haematoma at the back of the thigh. This seemed to resolve in a few weeks. Three years later a further attempt to correct the deformity with daily manual extension and active exercises failed, but a turnbuckle plaster and wedging in the same year gained correction in four months to 5 degrees short of full extension without apparent complications. The plaster was then removed and a caliper fitted. A prosthesis was supplied for the other limb, and five months later he was discharged, walking. He then led an active life for six years despite various haemorrhagic mishaps.

The first available radiographs, taken at the age of seventeen, showed changes in the left femur (Figs. 1 and 2) but at that time the leg appeared to be fairly normal in outline. Two years later, however, he was involved in a motor accident which by direct violence produced

* Formerly Nuffield Scholar, Nuffield Department of Orthopaedic Surgery, Oxford.

† Clinical Research Fellow, Medical Research Council Blood Coagulation Research Unit, the Churchill Hospital, Oxford. 


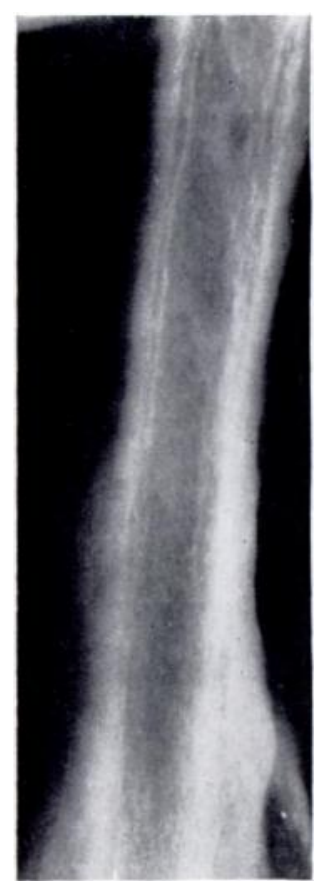

FIG. 1

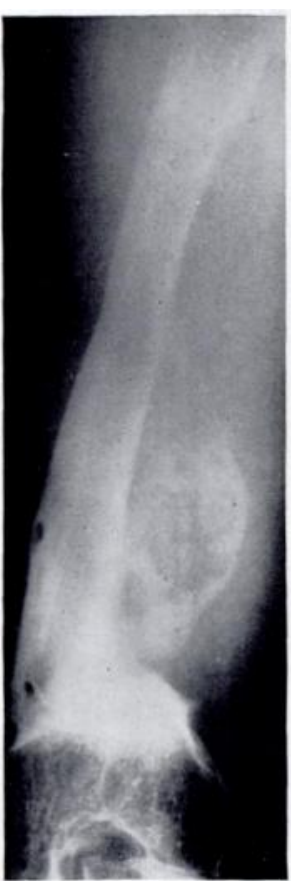

FIG. 2

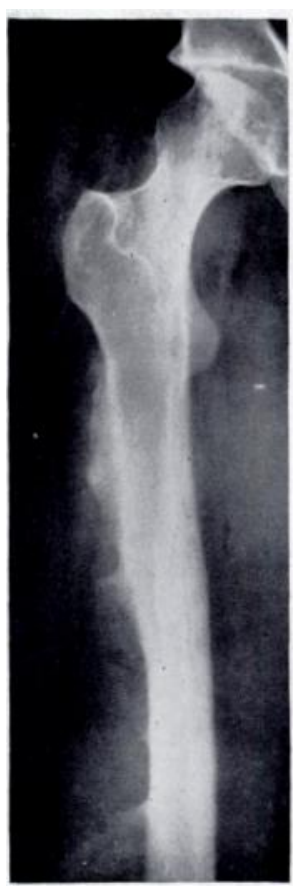

Fig. 3

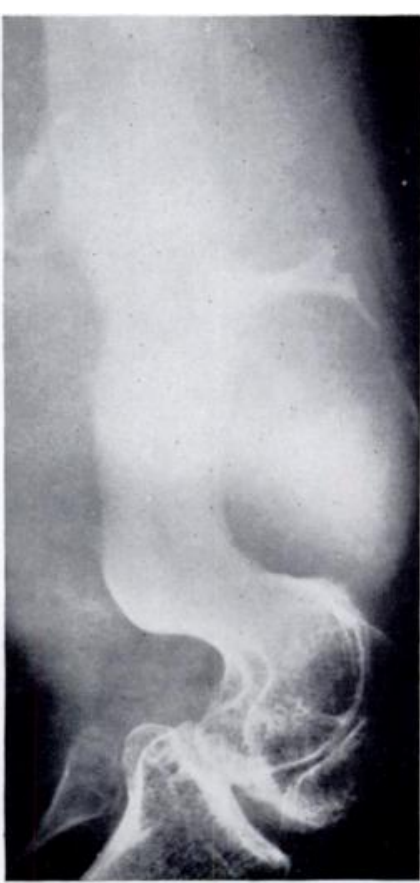

FIG. 4

Figures 1 and 2-Radiographs of the patient at the age of 17 years showing changes in the midshaft of the femur and in the lower femur. Figures 3 and 4-Radiographs of the patient at the age of 19 years which show calcification extending into the surrounding tissues from the upper femoral shaft and the lower femoral shaft.

a massive haemorrhage into the left thigh. From that time the leg never regained its former size, and although he continued his clerical work and had many other interests, he was unable to walk and relied completely on his wheelchair and invalid car.

At that time the circumference of the left thigh was three inches greater than that of the right, and radiographs suggested the appearance of a large calcified haematoma attached to the femur (Figs. 3 and 4). In the next four years the circumference of the limb had increased by a further eight inches, and amputation was suggested but refused. Subsequent radiographs showed progressive thinning and deformation of the shaft of the femur (Fig. 5).

By 1962 when he was twenty-four the limb had become a useless, massive and dangerous appendage (Fig. 6). The thigh measured thirty-four inches in circumference, and the risk of fracture of the femur, or of perforation of the skin with subsequent infection, or of spread of the tumour into the pelvis seemed imminent. When the hazards of allowing the cyst to develop

FIGS. 5 AND 6

Figure 5-A radiograph when the patient was 24 years showing thinning and deformation of the left femoral shaft. Figure 6-The appearance of the limb before amputation.

vol. 47 B, NO. 2, MAY 1965

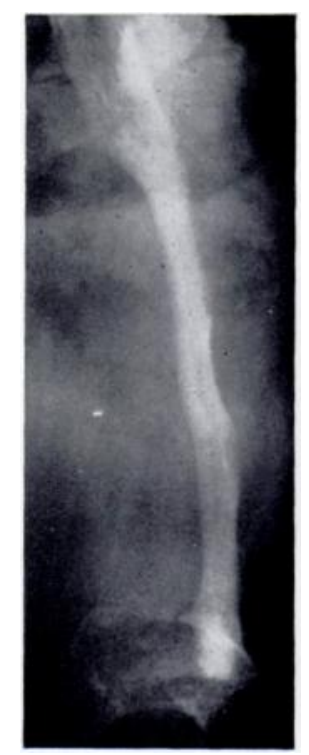

Fig. 5

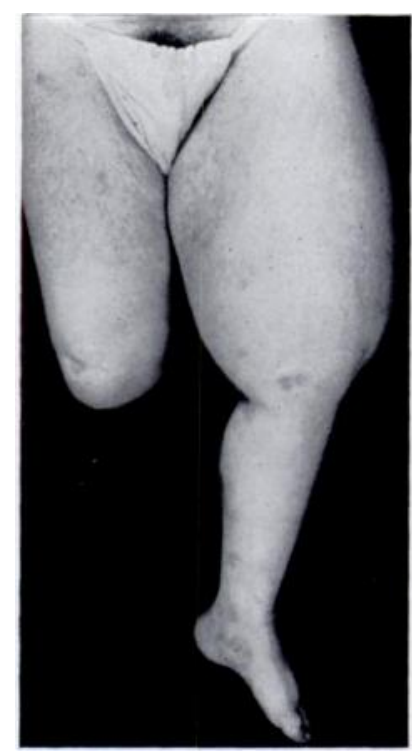

FIG. 6 


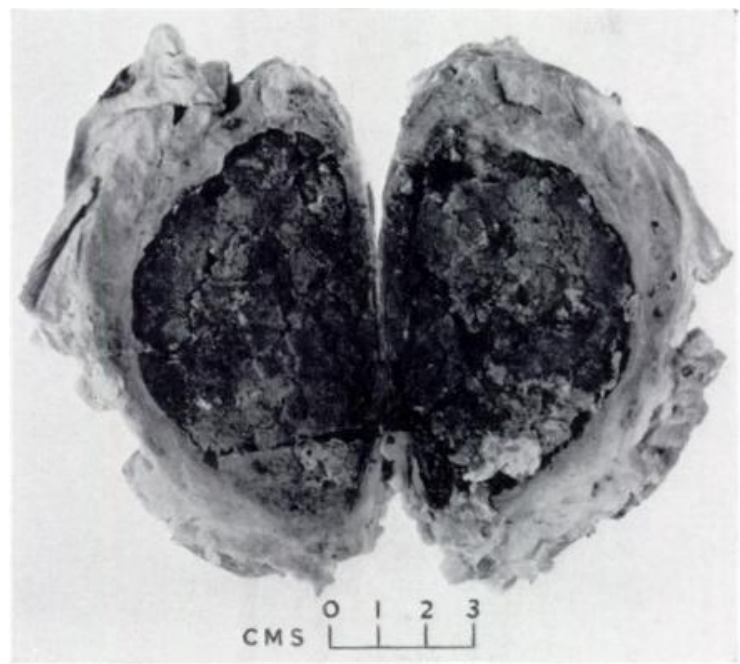

Fig. 7

A longitudinal section of the cystic haematoma in the biceps femoris muscle.

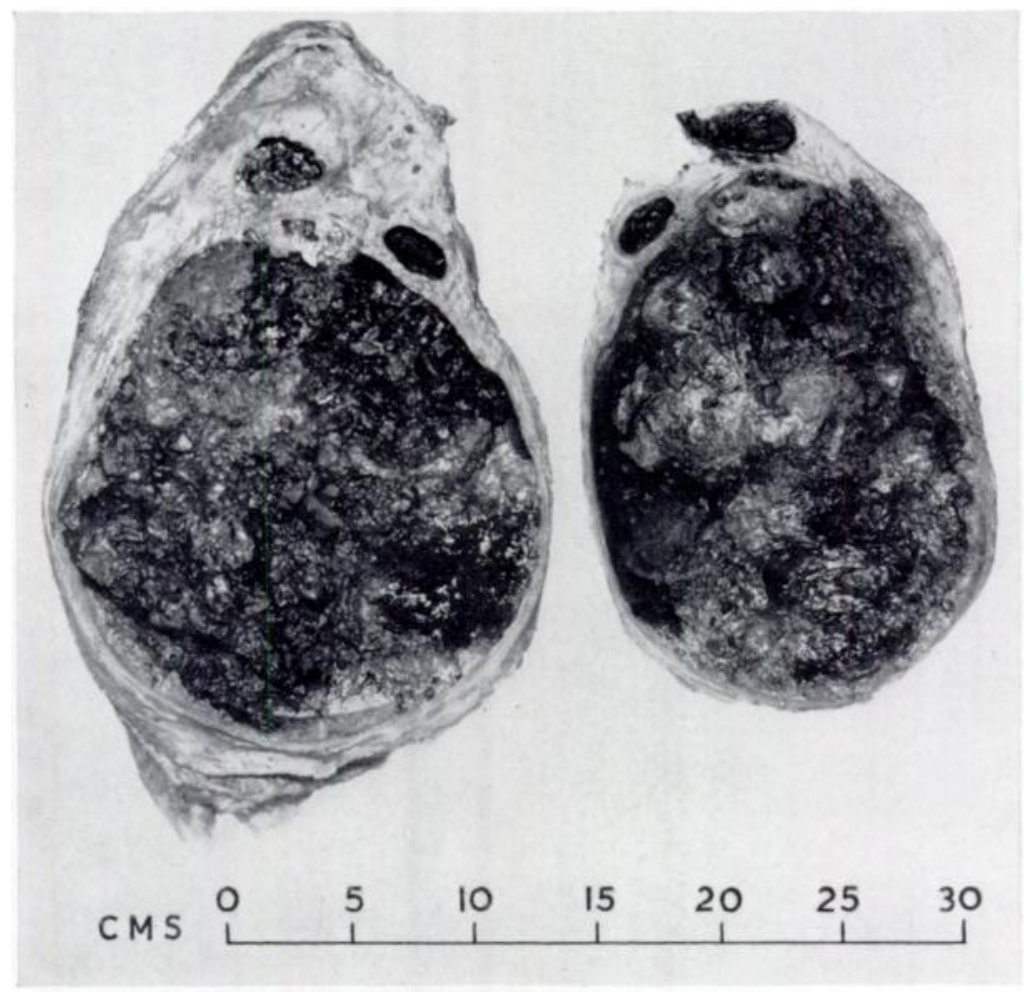

Fig. 8

A sagittal section of the main cyst of the thigh. 
further were explained to him and weighed against the dangers and possible outcome of operation he agreed to have the leg amputated, and he was admitted to the Nuffield Department of Surgery under the care of Professor P. R. Allison.

Operation-A haemostatic level of AHG was obtained before operation by the infusion of a sufficient amount of a commercial concentrate of pig plasma AHG. The superior and inferior venae cavae were then cannulated through the left cephalic and the right saphenous veins. AHG infusion was continued throughout the operation so that its level immediately after operation was 70 per cent of normal. The left iliac vessels were exposed through a vertical incision and occluded with a string and rubber tourniquet. The lower end of the vertical incision was extended in racket fashion around the upper end of the thigh. The femoral vessels were divided between sutures in the femoral triangle and the upper end of the femur was exposed.

There was no evidence of disease in the adductors or tensor fasciae latae, and no sign of spread into the pelvis. The upper limit of the tumour was formed by the insertion of the vastus lateralis, so the femur was divided at the level of the lesser trochanter.

Amputation was completed, and after careful haemostasis the tourniquet was released. The muscles were left unsutured and bleeding from bone controlled with bone wax. The total blood loss was about 1,500 millilitres and was replaced during operation. The early course after operation was uneventful. On the seventeenth day there was some gaping of the wound; about 200

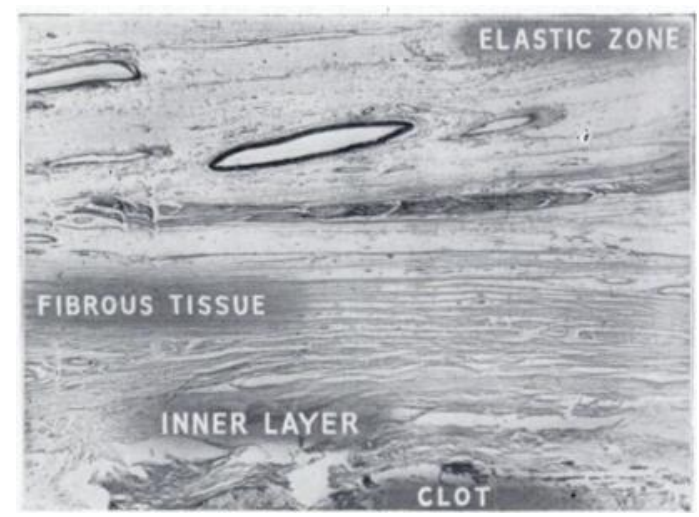

FiG. 9

The wall of the cyst showing three main zones, an inner one containing haemosiderin, a middle fibrous layer, and an outer reticular zone containing large vessels and elastic tissue. (Haematoxylin and eosin, $\times 15$.) millilitres of old blood clot were evacuated and the cavity was irrigated and lightly packed. Healing was complete eight weeks later. Besides obtaining an adequate level of AHG for the operation, daily infusions were continued for four weeks afterwards.

High potency animal concentrates were used for the first eighteen days, and then freshfrozen plasma or human AHG concentrate.

Progress has been satisfactory. He has good control of the stump and can walk on artificial limbs, although he finds these too traumatic for everyday use. He is working and has married. Pathology of the amputated limb-The weight of the leg was 51 pounds. Shortly after removal it was perfused with a 25 per cent colloidal barium sulphate suspension (Micropaque) through the superficial and deep femoral arteries, and fixed in 10 per cent formalin solution.

After removal of the skin a small opening was made in the lateral side of the wall of the mass. A dark fluid, not under pressure, oozed out. There was no communication with the knee joint, and the outer side of the wall seemed to be covered by the vastus lateralis and the aponeurotic part of the fascia lata. The flexor compartment of the thigh was identified, and most of the hamstring muscles were separated by dissection from the wall of the cyst. The biceps femoris contained a circumscribed hard fusiform mass measuring four inches in length by two and a half inches in diameter, which was found to be an encapsulated haematoma full of solid brownish unorganised clots (Fig. 7).

Most of the wall of the cyst was formed by the muscles of the extensor group, except on the medial side, where the distal two-thirds of the adductor muscles took part.

The patella was anchored by fibrous tissue to the femur. There were no signs of recent haemarthrosis, but there was fibrosis of the capsule and synovial membrane. The articular cartilage was thin, irregular and dull in appearance.

VOL. 47 B, NO. 2, MAY 1965

$D_{(4)}$ 


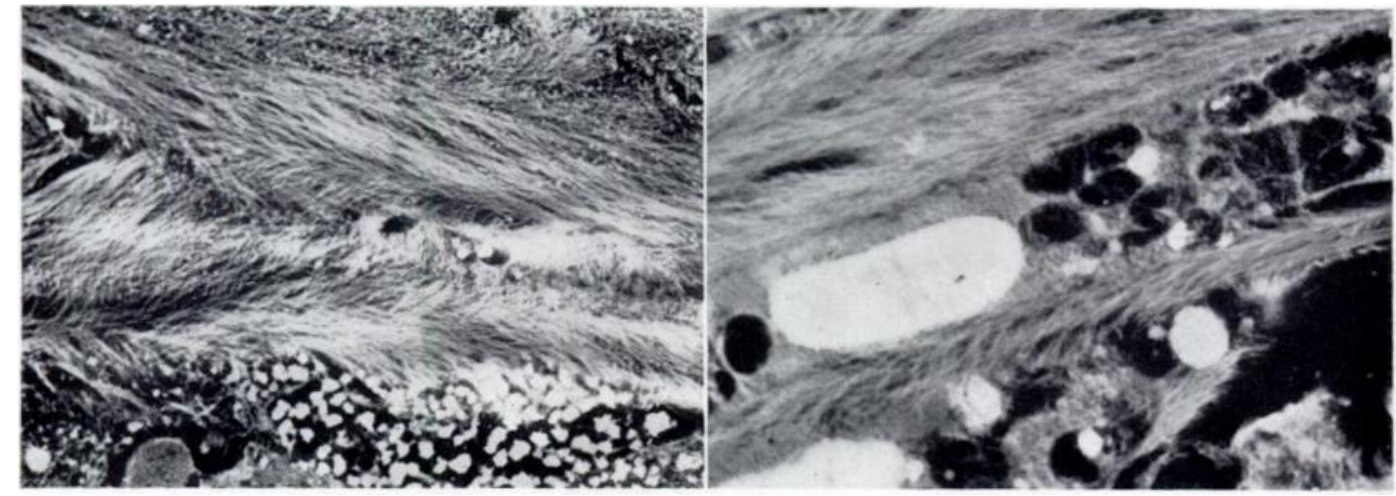

FIG. 10

FIG. 11

Figure 10-An electron micrograph showing a deposit of electron-dense haemosiderin granules, which appear as a white stippled area, surrounded by bands of fibrous collagen. From the inner cyst wall. $(\because 2,500$.) Figure 11-Electron micrograph of section of inner zone of cyst wall, showing two dense nuclei whose appearance suggests an active pre-division phase. $(\cdot 3,750$.)

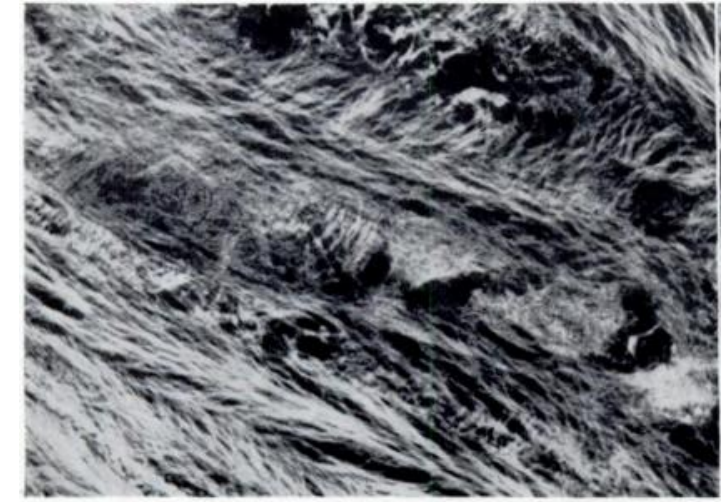

FIG. 12

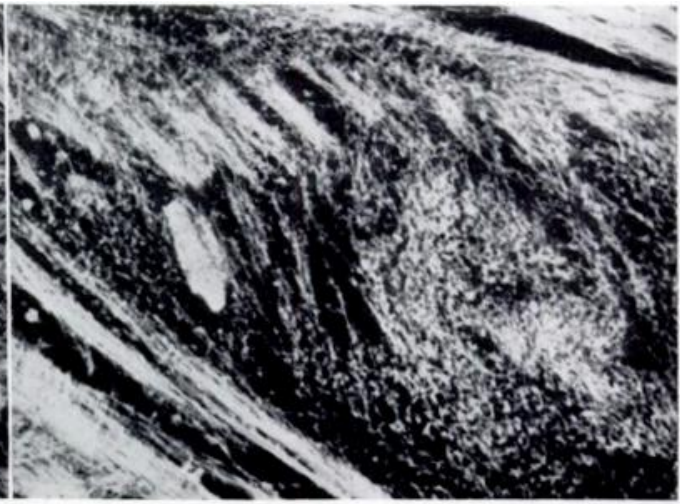

Fig. 13

Figure 12-This electron microscope photograph of part of a section of the main cyst wall shows a dense fibrous structure composed largely of collagen fibrils. $(\times 3,750$.) Figure 13 -Diffuse area of non-collagenous protein between dense bands of collagen. Electron micrograph. $(\times 2,500$.

The greatest bulge of the cyst was on the medial side. The whole cyst was divided longitudinally leaving the femur intact (Fig. 8). Inside the cyst was a solid mass of clot with a small amount of fluid. The clot was brownish and of putty-like consistency and contained a number of darker lumps of amorphous material. The wall of the haematoma was a tough membrane, from which the clot was easily separated by gentle scraping. After the clot had been removed the femur was clearly visible, forming a ridge on the posterior aspect of the cyst. There was no communication between the medial and lateral sides of the cyst behind the femur because the fibrous attachments to the linea aspera formed a firm barrier. The vascular perfusion medium did not penetrate to the haematoma or to the inner side of the wall.

In the medial wall of the cyst there were two fibrous struts, ossified at their inferior ends, where they formed a continuation of the lateral surface of the femoral cortex. A similar ridge of bony tissue was observed along the junction of the wall of the cyst with the femur. It was especially well developed at the distal end of the metaphysis, close to the intercondylar space. Most of the inner surface of the tumour, including the whole of the exposed surface of the femur, and the struts, was covered by a thin membrane. Small perfused vessels could be seen in some areas of this membrane. Periosteum was absent from the exposed area of bone. 


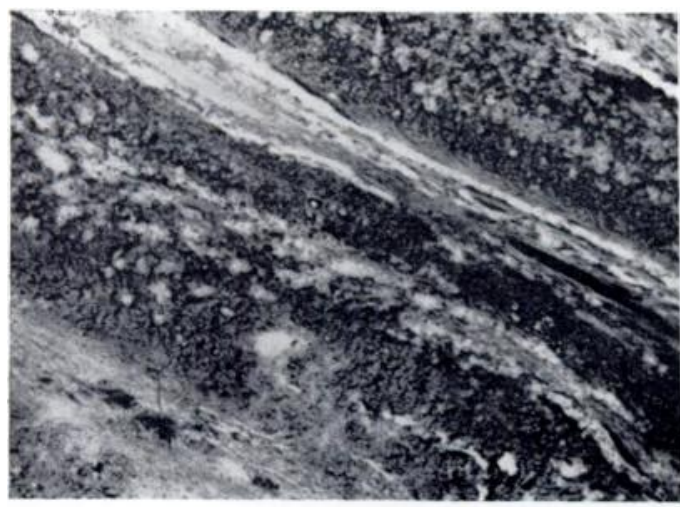

FIG. 14

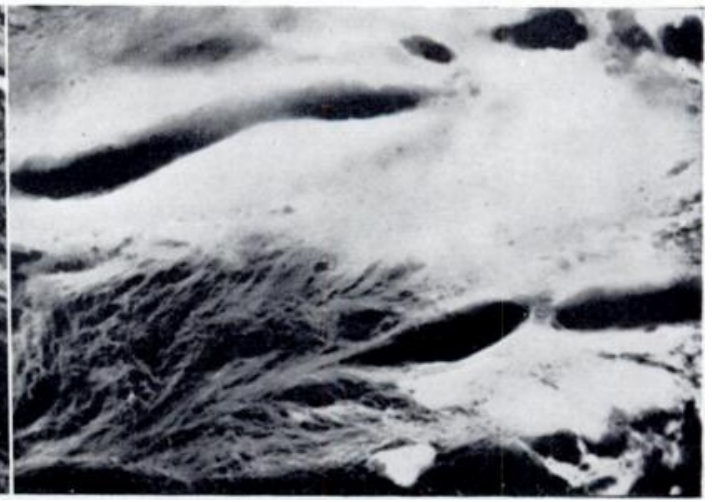

FIG. 15

Figure 14-Electron micrograph showing whiter elastic fibres cut in different directions. Section of junctional area between main cyst wall and outer zone. $(\times 2,500$.) Figure $15-$ Section of large elastic fibre from outer zone of cyst wall. Electron micrograph. $(\lessdot 2,500$.

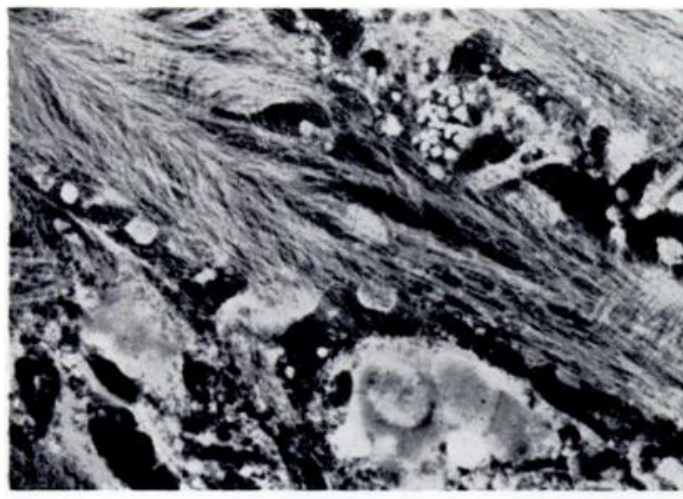

Fig. 16

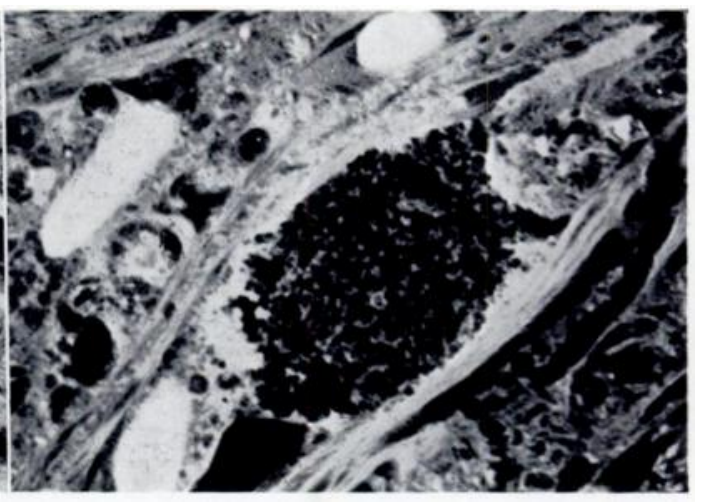

Fig. 17

Figure 16-Electron micrograph of a section of membrane which lines the cyst and covers exposed bone. This shows reticular collagenous structure with interposed scattered granules of haemosiderin, dense nuclei suggesting cell activity and some small capillaries. $(\times 2,500$.) Figure 17 -Electron micrograph of section of the lining membrane of the cyst showing a capillary containing granules of barium sulphate perfusion medium. The surrounding tissue contains several dense nuclei, suggesting cell activity. $(\times 2,500$.)

Two cystic areas, at the proximal end of the specimen, were found to be isolated intramuscular haematomata in the upper part of the vastus lateralis. They contained dark fluid but otherwise resembled the main cyst.

Histology - The haematoma consisted of masses of red blood cells at different stages of degeneration and lysis. Embedded in gelatinous clot were solid clumps of degenerate cells. Perl's stain showed some haemosiderin in the clot, particularly in the outer parts.

The wall of the cyst was composed of various layers of fibrous tissue, covered by strands of muscle fibres running in various directions. The fibrous capsule showed three main zones (Fig. 9). An inner thin layer contained more haemosiderin than elsewhere, some of it between the collagen strands (Fig. 10). ${ }^{*}$ Here, in some places, blood clot was incorporated into the inner layer by bands of collagen, whilst in other parts of the lining there was evidence of active cell proliferation which was sharply delimited from the clot (Fig. 11). Outside this

* Figures 10 to 17 are electron microscope photographs of specimens prepared by the method described for pathological material by Little (1961). Tissue blocks were fixed in neutral formalin then embedded in a mixture of butyl and isobutyl methacrylate which was polymerised for four hours at room temperature with ultra-violet light. Sections were cut and mounted, and the methacrylate was removed by solution in amyl acetate. The photographs are printed so that electron-dense structures are shown in white.

vOL. 47 B, NO. 2, MAY 1965

$$
\text { D-1 }(8)
$$


layer the main part of the cyst wall consisted of dense fibrous tissue (Fig. 12), with the collagen bundles interspersed with non-fibrillar material, probably a non-collagenous protein (Fig. 13). Strands of elastic tissue were apparent in the outer part of this layer (Fig. 14), but vessels were small and few in number. This was in distinct contrast to the outer fibrous zone, which was more reticular in structure and where both elastin fibres (Fig.15) and vessels were abundant and of large size. The thin membrane which covered exposed bone and part of the interior of the cyst wall showed poorly formed collagen strands with haemosiderin deposits between them (Fig. 16). Many of the cells possessed densely staining nuclei, indicating cell activity (Rigal and Little 1962), and there were a few small capillaries (Fig. 17).

Light and electron microscopy of sections of the inner cyst wall showed active cell proliferation and the presence of non-collagenous protein, which suggested that the poor collagen formation resulted from expansion of the cyst, and from the intermittent pressures to which the wall of the cyst was submitted between its contents and the surrounding muscles.

Sections of the bone and the adjoining cyst wall showed that the periosteum, which was present on bone outside the cyst, continued for a short distance in the cyst wall but was absent from the surface of the bone inside the cyst. This suggested that the periosteum had been stripped off the bone during the formation of the cyst.

\section{DISCUSSION}

It is significant that of twenty-two cases of "pseudotumour" reported in the literature thirteen were in the thigh, five were in the abdomen and pelvis, three in the tibia, and one (Fraenkel, Taylor and Richards 1959) had bilateral involvement of the thighs and both sides

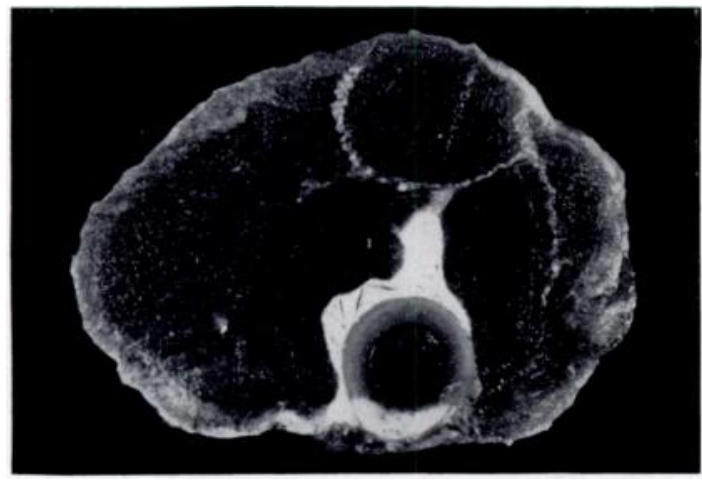

FIG. 18

Transverse section of mid-thigh of dog showing anterior periosteal elevation by injected opaque material. of the pelvis. They are mainly related to the powerful muscle groups of the iliopsoas, quadriceps, triceps surae and gluteus maximus. All of these muscles have profuse vascular connections with the underlying periosteum and bone, and all have firm connections between their muscle fibres and the periosteum, but not to any great extent with the bone itself. During growth the periosteum can be easily detached from the underlying cortex, particularly on the anterior surfaces of the ilium, femur and tibia. Also, these sites are all subject to the effect of gravity in the erect position, which would promote haemorrhage from damaged vessels.

Experiment-An experiment was done to show the immediate result of a subperiosteal bleed. A screw-tapped needle was passed through the femur of a young dog so as to emerge under the opposite periosteum. Injected material made a clean dissection of the periosteum and muscle in the plane of the shaft of the bone (Fig. 18). Such a separation might follow a subperiosteal haemorrhage, whatever the original cause. If intermittent pulling by the attached muscle fibres were combined with poor haemostasis and lack of organisation of the haematoma to prevent periosteal reattachment, further bleeding would be facilitated.

Figure 19 is a diagram of a transverse section of a human thigh to show the relationship of the extensor group of muscles to the femoral shaft. This is the area occupied by the cystic haematoma described in this paper, where posterior communication is prevented by the aponeurotic structures attached to the linea aspera, and where, moreover, the bare area of bone exposed in the cyst is that of the periosteal attachment of one muscle, the vastus intermedius. 
The bone changes which occurred in this patient seem a logical consequence of a lifting of the periosteum and are similar to those experimentally demonstrated by Trueta and Cavadias in 1955. There is loss of the outer third of the cortex with new bone formation in the inner layer of the periosteum. This may be seen radiologically.

Ossification of small subperiosteal haematomas is a well recognised phenomenon. Large subperiosteal haematomas are rare, but as early as 1893 van Arsdale described the formation of a large ossifying subperiosteal haematoma after a violent injury to the right humerus in a healthy twenty-two-year-old man.

Intraosseous haemorrhage as the initial cause of haemophilic pseudotumour has been suggested by Ivins (1957) and Horwitz, Simon and Bassen (1959). Silber and Christensen (1959) and Abell and Bailey (1960) found a basis for this hypothesis in Larsen's experimental demonstration of medullary necrosis by increasing the intramarrow pressure (1938), but this experiment produced necrosis of the marrow and of the inner cortex of the bone. If as in osteomyelitis the pressure is continued, the cortex is perforated, the periosteum is elevated and bone necrosis occurs. Descriptions of the large haematomata in haemophiliacs do not suggest resorption of the inner part of the cortex or bone necrosis.

There are other observations which seem to exclude the possibility of the initial lesion being an intraosseous haemorrhage. Experience gained at centres familiar with this problem has shown that fractures and osteotomies in severe haemophiliacs heal as readily as those in normal individuals, and, while a high degree of antihaemophilic treatment is required for the soft-tissue operation involved, very little cover may be needed for the bone injury. This is also apparent in the management of closed fractures, which require plasma for only a day or two to aid resolution of tissue

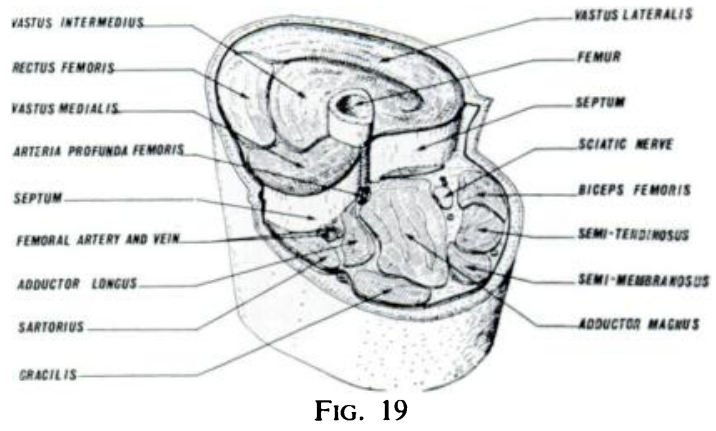

Diagram of transverse section of middle of human thigh to demonstrate the relationship of the extensor group of muscles to the femoral shaft. haematomata and then pursue a normal course. It is significant that the endosteal callus develops normally, while there is an almost complete absence of periosteal callus. Any intraosseous haemorrhage might therefore be expected to disperse in a normal manner.

Subperiosteal haematoma of the femur resulting from haemarthrosis of the knee has been reported by Reinecke and Wohlwill (1929). In the present case there was no apparent communication between the cyst and the adjacent joints.

It is possible that a small subperiosteal haematoma was formed, at the time when the knee flexion deformity was corrected, by a small fracture of the distal femoral metaphysis. Unfortunately there are no radiographic records of the affected femur until five years later, when bone changes were seen all along the femoral shaft. During the next two years, and after further injury to the thigh, the cyst enlarged and bone formation in the walls could be seen clearly, especially near the femoral shaft (Figs. 3 and 4). The subsequent progressive enlargement of the haematoma left struts in the wall of the cyst and produced some resorption of bone with further thinning of the diaphysis of the femur, so that a fracture seemed imminent.

Large intramuscular haematomata may produce bone resorption by interruption of the blood supply to the adjacent periosteum and outer cortex. The cases reported by Fraenkel et al. (1959) and those of Hall, Handley and Webster (1962) are good examples of this type of haemophilic cyst. We have seen three similar cases in whom no bony lesion was shown radiologically.

We believe that there are three main types of haemophilic cyst involving muscle which may be defined by their mode of origin and subsequent course: 1) The simple cyst, which occurs

VOl. 47 B, NO. 2, MAY 1965 
within the fascial envelope of a muscle or muscles and which is confined by the tendinous attachments. It may remain localised or it may track between muscle and fascia to point internally or through the skin. There are no radiological changes. The cyst in the biceps femoris of this patient is a good example of this type. 2) The cyst which develops in a muscle with wide fibrous periosteal attachments may progress to give rise to cortical thinning because it interferes with the periosteal blood supply. The patients of Fraenkel and of Hall fit this category. 3) The pseudotumour which starts as a subperiosteal haemorrhage with stripping of the periosteum from the cortex until this is limited by aponeurotic or tendinous attachments, and which then raises or destroys the muscles.

The hazards of aspiration, biopsy or evacuation of encapsulated haematomata are apparent throughout the literature. Needle tracks and surgical defects of the fibrous cyst wall fail to heal, the cysts refill with blood and become infected; surgical wounds break down or form fistulae. The cyst wall is unable to form adequate granulation tissue for permanent collapse and healing.

It may be possible to manage the simple cyst or early subperiosteal haematoma conservatively by haemostatic infusions and immobilisation. When changes are advanced, with periosteal elevation, bone involvement and a history of steady progression, the only chance of complete cure is given by a radical approach, either by enucleation or selective amputation. The management of major surgical operations in haemophilia has been discussed by Macfarlane, Biggs and Bidwell (1954), Macfarlane, Mallam, Witts, Bidwell, Biggs, Fraenkel, Honey and Taylor (1957), Fraenkel and Honey (1955), Fraenkel (1957) and Hall ct al. (1962).

\section{SUMMARY}

1. The case history of a haemophiliac in whom a large haematoma of the thigh was treated by amputation of the limb is described.

2. Examination of the available radiographs and of the histology led to the conclusion that the cyst was subperiosteal in origin.

3. Evidence is presented to support the hypothesis that haemophilic pseudotumours are always associated with subperiosteal haemorrhage, and that those haemophilic cysts which are confined to muscle have little or no effect on the adjacent bone.

Thanks are due to Professor J. Trueta and Professor R. G. Macfarlane for advice and encouragement; Professor P. R. Allison for permission to publish details of the operation; $\mathrm{Dr} \mathrm{K}$. Little for taking the electron microssope photographs; Dr L. Munuera for the drawing for Figure 19; and to Mr D. Drury for the illustrations.

\section{REFERENCES}

Abell, J. M., Jun., and Bailey, R. W. (1960): Hemophilic Pseudotumor. Archives of Surgery, 81, 569.

van Arsdale, W. W. (1893): Ossifying Haematoma. Annals of Surgery, 18, 8.

Crock, H. V., and BoNi, V. (1960): The Management of Orthopaedic Problems in Haemophiliacs. British Journal of Surgery, 48, 8.

Echternacht, A. P. (1943): Pseudotumor of Bone in Hemophilia. Radiology, 41, 565.

Fraenkel, G. J. (1957): Surgery in Haemophilia. Journal of the Royal College of Surgeons of Edinburgh, 3, 54.

Fraenkel, G. J., and Honey, G. E. (1955): Gunshot Wounds in a Haemophilic Patient. Successful Treatment by Animal Antihaemophilic Globulin and Surgery. Lancet, ii, 1117.

Fraenkel, G. J., Taylor, K. B., and Richards, W. C. D. (1959): Haemophilic Blood Cysts. British Journal of Surgery, 46, 383.

Hall, M. R. P., Handley, D. A., and Webster, C. U. (1962): The Surgical Treatment of Haemophilic Blood Cysts. Journal of Bone and Joint Surgery, 44-B, 781.

Horwitz, H., Simon, N., and Bassen, F. A. (1959): Haemophilic Pseudotumour of the Pelvis. British Journal of Radiology, N.S. 32, 51.

Ivins, J. C. (1957): Bone and Joint Complications of Hemophilia. In Hemophilia and Hemophilioid Diseases; International Symposium, p. 225. Edited by K. M. Brinkhous. Chapel Hill, N.C.: University of North Carolina Press. 
LARSEN, R. M. (1938): Intramedullary Pressure with Particular Reference to Massive Diaphyseal Bone Necrosis. Annals of Surgery, 108, 127.

LitTle, K. (1961): Use of Electron Microscope in Examination of Pathological Conditions Affecting Hard and Connective Tissues. Journal of the Royal Microscopical Society, 80, 33.

Macfarlane, R. G., Biggs, R., and Bidwell, E. (1954): Bovine Antihaemophilic Globulin in the Treatment of Haemophilia. Lancet, i, 1316.

Macfarlane, R. G., Mallam, P. C., Witts, L. J., Bidwell, E., Biggs, R., Fraenkel, G. J., Honey, G. E., and TAYLOR, K. B. (1957): Surgery in Haemophilia. The Use of Animal Antihaemophilic Globulin and Human Plasma in Thirteen Cases. Lancet, ii, 251.

Reinecke and WoHlwill. (1929): Über hämophile Gelenkerkrankung. Archiv fïr Klinische Chirurgie, 154, 425.

Rigal, W. M., and Little, K. (1962): Some Observations on Nuclear Structure in Cartilage Cells. Journal of the Royal Microscopical Society, 80, 279.

Silber, R., and Christensen, W. R. (1959): Pseudotumor of Hemophilia in a Patient with PTC Deficiency. Blood, 14, 584.

Starker, L. (1918): Knochenusur durch ein hämophiles subperiostales Hämatom. Mitteilungen aus den Grenzgebieten der Medizin und Chirurgie, 31, 381.

Trueta, J., and Cavadias, A. X. (1955): Vascular Changes Caused by the Küntscher Type of Nailing. Journal of Bone and Joint Surgery, 37-B, 492.

VOL. 47 B, NO. 2, MAY 1965

D-2 (4) 